\title{
The dog-and-rabbit chase problem as an exercise in introductory kinematics
}

\author{
O. I. Chashchina ${ }^{1}$, Z. K.Silagadze ${ }^{1,2}$ \\ ${ }^{1}$ Department of Physics, Novosibirsk State University, 630 090, Novosibirsk, Russia. \\ ${ }^{2}$ Budker Institute of Nuclear Physics, 630 090, Novosibirsk, Russia. \\ E-mail: silagadze@inp.nsk.su
}

(Received 30 August 2009, accepted 19 September 2009)

\begin{abstract}
The purpose of this article is to present a simple solution of the classic dog-and-rabbit chase problem which emphasizes the use of concepts of elementary kinematics and, therefore, can be used in introductory mechanics course. The article is based on the teaching experience of introductory mechanics course at Novosibirsk State University for first year physics students which are just beginning to use advanced mathematical methods in physics problems. We hope it will be also useful for students and teachers at other universities too.
\end{abstract}

Keywords: Physics Education, Classical Mechanics teaching.

\section{Resumen}

El propósito de este artículo es presentar una solución simple al problema clásico del perro y el conejo, el cual remarca el uso de los conceptos básicos de cinemática y por lo tanto puede ser empleado en un curso introductorio de Mecánica. Este artículo está basado en la experiencia generada del curso introductorio de Mecánica en la Universidad Estatal de Novosibirsk para el primer año de Física dónde los estudiantes comienzan con métodos avanzados de matemáticas en los problemas de Física. Se espera que el presente trabajo sea útil tanto para los estudiantes como para los alumnos y otras universidades también.

Palabras clave: Educación en Física, Enseñanza de la Mecánica Clásica.

PACS: 01.40.Fk, 45.20.D-

ISSN 1870-9095

\section{INTRODUCTION}

A rabbit runs in a straight line with a speed $u$. A dog with a speed $V>u$ starts to pursuit it and during the pursuit always runs in the direction towards the rabbit. Initially the rabbit is at the origin while the dog's coordinates are $x(0)=0, y(0)=$ $L$ (see Fig. 1). After what time does the dog catch the rabbit?

This classic chase problem and its variations are often used in introductory mechanics course [1, 2, 3]. When one asks to find the dog's trajectory (curve of pursuit), the problem becomes an exercise in advanced calculus and/or in the elementary theory of differential equations [4, 5]. However, its treatment simplifies if the traditional machinery of physical kinematics is used [6].

The mathematics of the solution becomes even simpler if we further underline the use of physical concepts like reference frames, vector equations, decomposition of velocity into radial and tangential components.

\section{DURATION OF THE CHASE}

Let $\vec{r}_{1}$ be a radius-vector of the dog and $\vec{r}_{2}-$ a radius-vector of the rabbit. So that

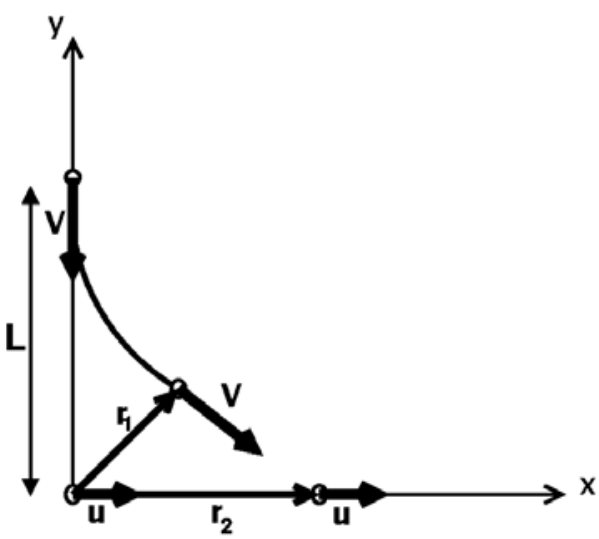

FIGURE 1. Dog and rabbit chase. The dog is heading always towards the rabbit.

$$
\dot{\vec{r}}_{1}=\vec{V}, \quad \dot{\vec{r}}_{2}=\vec{u}
$$

As the dog always is heading towards the rabbit, we can write

$$
\vec{r}_{2}-\vec{r}_{1}=k(t) \vec{V} .
$$




\section{O. I. Chashchina and Z. K.Silagadze}

The proportionality coefficient $k$ depends on time. Namely, at the start and at the end of the chase we, obviously, have

$$
k(0)=\frac{L}{V}, \quad k(T)=0 .
$$

Differentiating (2) and using (1), we get

$$
\vec{u}-\vec{V}=\dot{k}(t) \vec{V}+k(t) \dot{\vec{V}}
$$

As the dog's velocity does not change in magnitude, it must be perpendicular to the dog's acceleration all the time:

$$
\vec{V} \cdot \dot{\vec{V}}=0
$$

Formally this follows from

$$
0=\frac{d V^{2}}{d t}=\frac{d \vec{V}^{2}}{d t}=2 \vec{V} \cdot \dot{\vec{V}}
$$

Equations (4) and (5) imply

$$
\vec{V} \cdot(\vec{u}-\vec{V})=\dot{k}(t) V^{2},
$$

or

$$
u V_{x}-V^{2}=\dot{k}(t) V^{2}
$$

We can integrate the last equation and get

$$
u x-V^{2} t=k(t) V^{2}-L V
$$

At the time $t=T$, when the dog catches the rabbit and the chase terminates, we must have $x=u T$ and remembering that $k(T)=0$ we easily find $T$ from (7):

$$
T=\frac{L V}{V^{2}-u^{2}}
$$

\section{DOG'S TRAJECTORY IN THE RABBIT'S FRAME}

Let us decompose the dog's velocity into the radial and tangential components in the rabbit's frame (see Fig. 2):

$$
\begin{aligned}
& V_{r}=-V+u \cos (\pi-\varphi)=-V-u \cos \varphi \\
& V_{\varphi}=u \sin (\pi-\varphi)=u \sin \varphi
\end{aligned}
$$

But $V_{r}=\dot{r}$ and $V_{\varphi}=r \dot{\varphi}$. Therefore

$$
\dot{r}=-V-u \cos \varphi, \quad r \dot{\varphi}=u \sin \varphi
$$

If we divide the first equation on the second and take into account that

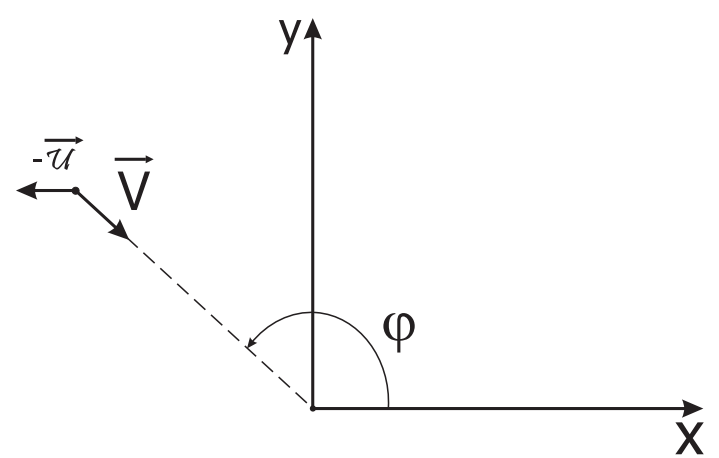

FIGURE 2. Components of the dog's velocity in the rabbit's frame.

$$
\frac{\dot{r}}{\dot{\varphi}}=\frac{d r}{d \varphi}
$$

we get

$$
\frac{1}{r} \frac{d r}{d \varphi}=-\frac{V+u \cos \varphi}{u \sin \varphi}
$$

Hence

$$
\ln \frac{r}{L}=-\int_{\pi / 2}^{\varphi} \frac{V+u \cos \varphi}{u \sin \varphi} d \varphi
$$

Putting $Z=\cos \varphi$ and using the decomposition

$$
\frac{\frac{V}{u}+z}{1-z^{2}}=\frac{A}{1-z}+\frac{B}{1+z},
$$

where

$$
A=\frac{1}{2}\left(1+\frac{V}{u}\right), \quad B=\frac{1}{2}\left(\frac{V}{u}-1\right),
$$

we can easily integrate and get

$$
-\int_{\pi / 2}^{\varphi} \frac{V+u \cos \varphi}{u \sin \varphi} d \varphi=\ln \frac{(1+\cos \varphi)^{B}}{(1-\cos \varphi)^{A}}=\ln \left[\left(\operatorname{ctg} \frac{\varphi}{2}\right)^{\frac{V}{u}} \frac{1}{\sin \varphi}\right] .
$$

Therefore, the equation of the dog's trajectory in the rabbit's frame is

$$
r=\frac{L}{\sin \varphi}\left(\operatorname{ctg} \frac{\varphi}{2}\right)^{\frac{v}{u}}
$$

\section{DOG'S CURVE OF PURSUIT}

Let us now find the dog's trajectory in the laboratory frame. From (9) and (10), we get 


$$
\frac{L \dot{\varphi}}{\sin \varphi}\left(\operatorname{ctg} \frac{\varphi}{2}\right)^{\frac{V}{u}}=u \sin \varphi,
$$

which, by using

$$
\frac{d \varphi}{\sin ^{2} \varphi}=-\frac{1}{2}\left[1+\frac{1}{\operatorname{ctg}^{2} \frac{\varphi}{2}}\right] d\left(\operatorname{ctg} \frac{\varphi}{2}\right)
$$

can be recast in the form

$$
\left[1+\frac{1}{\operatorname{ctg}^{2} \frac{\varphi}{2}}\right]\left(\operatorname{ctg} \frac{\varphi}{2}\right)^{\frac{v}{u}} d\left(\operatorname{ctg} \frac{\varphi}{2}\right)=-\frac{2 u}{L} d t .
$$

Therefore

$$
\frac{t}{T}=1-\frac{1}{2 v}\left[(v-1)\left(\operatorname{ctg} \frac{\varphi}{2}\right)^{v+1}+(v+1)\left(\operatorname{ctg} \frac{\varphi}{2}\right)^{v-1}\right],
$$

where

$$
v=\frac{V}{u},
$$

and $T$ was defined earlier through (8). But by (10)

$$
y=r \sin \varphi=L\left(\operatorname{ctg} \frac{\varphi}{2}\right)^{v},
$$

and, therefore, (11) reproduces the result of Ref. [6]

$$
\frac{t}{T}=1-\frac{1}{2}\left[(1-\tilde{v})\left(\frac{y}{L}\right)^{\tilde{v}+1}+(1+\tilde{v})\left(\frac{y}{L}\right)^{1-\tilde{v}}\right],
$$

where

$$
\tilde{v}=\frac{1}{v}=\frac{u}{V}
$$

From (7), we have

$$
\frac{x}{L}=\frac{v^{3}}{v^{2}-1}\left(\frac{t}{T}+\frac{k(t)}{T}\right)-v
$$

and to get the equation of the dog's trajectory, we have to express $k(t)$ through $y$. Taking the $y$ component of the vector equation (2), we get

$$
-y=k(t) \dot{y},
$$

The dog-and-rabbit chase problem as an exercise in introductory kinematics and therefore

$$
k(t)=-\frac{y}{\dot{y}} .
$$

It remains to differentiate (12) to get the derivative of $y$ and hence the desired expression for $k(t)$ :

$$
\frac{k(t)}{T}=-\frac{1}{2}\left(\tilde{v}^{2}-1\right)\left[\left(\frac{y}{L}\right)^{1+\tilde{v}}+\left(\frac{y}{L}\right)^{1-\tilde{v}}\right],
$$

Substituting (12) and (15) into (13), we finally get the dog's curve of pursuit in the form

$$
\frac{x}{L}=\frac{\tilde{v}}{1-\tilde{v}^{2}}+\frac{1}{2}\left[\frac{1}{1+\tilde{v}}\left(\frac{y}{L}\right)^{1+\tilde{v}}-\frac{1}{1-\tilde{v}}\left(\frac{y}{L}\right)^{1-\tilde{v}}\right] .
$$

Of course, this result is the same as found earlier in the literature $[4,5,6]$, up to applied conventions.

\section{THE LIMIT DISTANCE FOR EQUAL VELO- CITIES}

Let speeds of the dog and the rabbit are equal in magnitude and their initial positions are as shown in the Fig. 3. To what limit converges the distance between them?

It is convenient to answer this question in the rabbit's frame. For equal velocities, $V=u$, equations (9) take the form

$$
\dot{r}=-u(1+\cos \varphi), \quad r \dot{\varphi}=u \sin \varphi
$$

Therefore

$$
\frac{d}{d t}(r-r \cos \varphi)=\dot{r}(1-\cos \varphi)+r \dot{\varphi} \sin \varphi=0 .
$$

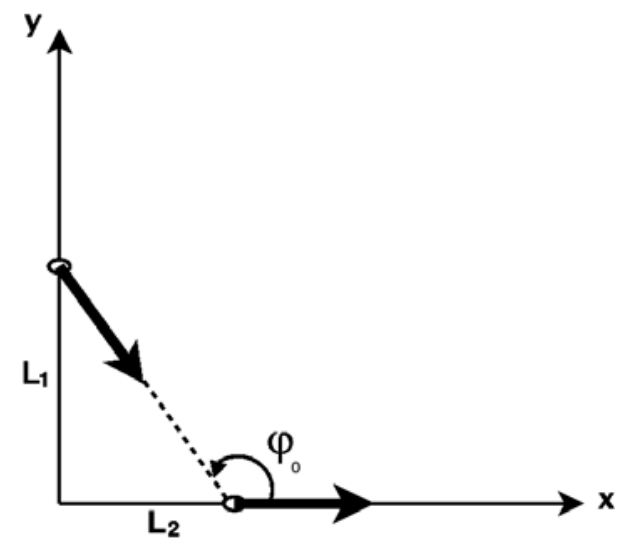

FIGURE 3. The chase with equal velocities. 


\section{O. I. Chashchina and Z. K.Silagadze}

We see that

$$
r(1-\cos \varphi)=C
$$

where $C$ is a constant. At $t=0$ we have (see Fig. 3)

$$
r_{0}=\sqrt{L_{1}^{2}+L_{2}^{2}}, \quad \cos \varphi_{0}=-\frac{L_{2}}{\sqrt{L_{1}^{2}+L_{2}^{2}}}
$$

Therefore

$$
C=L_{2}+\sqrt{L_{1}^{2}+L_{2}^{2}}
$$

In the rabbit's frame $\varphi \rightarrow \pi$, when $t \rightarrow \infty$. Hence the limit distance between the dog and the rabbit is [5]

$$
r_{\infty}=\frac{C}{1-\cos \pi}=\frac{L_{2}+\sqrt{L_{1}^{2}+L_{2}^{2}}}{2} .
$$

\section{CHASE ALONG THE TRACTRIX}

If the rabbit is allowed to change the magnitude of his speed, he can manage to keep the distance between him and the dog constant. Let us find the required functional dependence $u(t)$ and the dog's trajectory in this case. If $r=L=$ const, so that $\dot{r}=0$, equations (9) take the form

$$
V=-u \cos \varphi, \quad L \dot{\varphi}=u \sin \varphi
$$

Therefore

$$
\frac{L}{V} \dot{\varphi}=-\operatorname{tg} \varphi,
$$

which can be easily integrated to get

$$
\ln \left(\frac{\sin \varphi}{\sin \varphi_{0}}\right)=-\frac{V}{L} t
$$

or

$$
\sin \varphi=\sin \varphi_{0} \cdot e^{-\frac{V}{L} t}
$$

Then the first equation of (17) determines the required form of the rabbit's velocity

$$
u(t)=\frac{V}{\sqrt{1-\sin ^{2} \varphi_{0} \cdot e^{-\frac{V}{L} t}}},
$$

(note that we are assuming $\varphi \geq \varphi_{0}>\pi / 2$, as in Fig. 3, so that $\cos \varphi=-\sqrt{1-\sin ^{2} \varphi}$ ). Now let us find the dog's trajectory. We have (see Fig. 3)

$$
\begin{aligned}
x=\int_{0}^{t} u(\tau) d \tau-L \cos (\pi-\varphi) & =\int_{0}^{t} u(\tau) d \tau+L \cos \varphi \\
y=L \sin (\pi-\varphi) & =L \sin \varphi
\end{aligned}
$$

But

$$
\int_{o}^{t} u(\tau) d \tau=L \int_{\varphi_{0}}^{\varphi} \frac{d \varphi}{\sin \varphi}=-L \int_{\varphi_{0}}^{\varphi} \frac{d \cos \varphi}{1-\cos ^{2} \varphi},
$$

because

$$
u(t) d t=\frac{L}{\sin \varphi} d \varphi
$$

according to the second equation of (17).

By using the decomposition

$$
\frac{1}{1-\cos ^{2} \varphi}=\frac{1}{2}\left[\frac{1}{1+\cos \varphi}+\frac{1}{1-\cos \varphi}\right],
$$

the integral in (19) is easily evaluated with the result

$$
\int_{0}^{t} u(\tau) d \tau=L\left[\ln \left(\operatorname{ctg} \frac{\varphi_{0}}{2}\right)-\ln \left(\operatorname{ctg} \frac{\varphi}{2}\right)\right]
$$

Therefore the parametric form of the dog's trajectory is

$$
\frac{x}{L}=\cos \varphi+\ln \left(\operatorname{ctg} \frac{\varphi_{0}}{2}\right)-\ln \left(\operatorname{ctg} \frac{\varphi}{2}\right), \quad \frac{y}{L}=\sin \varphi .
$$

To get the explicit form of the trajectory, we use

$$
\begin{gathered}
\cos \varphi=-\sqrt{1-\frac{y^{2}}{L^{2}}}, \\
\operatorname{ctg} \frac{\varphi}{2}=\frac{1+\cos \varphi}{\sin \varphi}=\frac{L-\sqrt{L^{2}-y^{2}}}{y},
\end{gathered}
$$

which gives

$$
x=L \ln \left(\operatorname{ctg} \frac{\varphi_{0}}{2}\right)-L \ln \frac{L-\sqrt{L^{2}-y^{2}}}{y}-\sqrt{L^{2}-y^{2}}
$$

This trajectory is a part of tractrix - the famous curve [7, 8] with the defining property that the length of its tangent, between its directrix (the $x$-axis in our case) and the point of tangency, has the same value $L$ for all points of the tractrix. 


\section{CONCLUDING REMARKS}

We think the problem considered is of pedagogical value for undergraduate students, which take their first year course in physics. It demonstrates the use of some important concepts of physical kinematics, as already stressed by Mungan in Ref. [6]. The approach presented in this article requires only minimal mathematical background and, therefore, is suitable for students which just begin their physics education. However, if desired, this classic chase problem allows a demonstration of more elaborate mathematical concepts like Frenet-Serret formulas [9], Mercator projection in cartography [10], and even hyperbolic geometry (which is realized on the surface of revolution of a tractrix about its directrix) [11]. Interested reader can find some other variations of this chase problem in $[12,13,14,15,16]$.

\section{ACKNOWLEDGEMENTS}

The work is supported in part by grants Sci.School905.2006.2 and RFBR 06-02-16192-a.

\section{REFERENCES}

[1] Irodov, I. E., Problems in General Physics (NTTS Vladis, Moscow, 1997), p. 9 (in Russian).

[2] Belchenko, Yu. I., Gilev, E. A. and Silagadze, Z. K., Problems in mechanics of particles and bodies. Part 1: relativistic mechanics (Novosibirsk University Press, Novosibirsk, 2006), p. 9 (in Russian).
The dog-and-rabbit chase problem as an exercise in introductory kinematics

[3] Silagadze, Z. K., Test problems in mechanics and special relativity, Preprint physics/0605057.

[4] Olchovsky, I. I., Pavlenko, Yu. G. and Kuzmenkov, L. S., Problems in theoretical mechanics for physicists (Moscow University Press, Moscow, 1977), p. 9 (in Russian).

[5] Pták, P. and Tkadlec, J., The dog-and-rabbit chase revisited, Acta Polytechnica 36, 5-10 (1996).

[6] Mungan, C. A., A classic chase problem solved from a physics perspective, Eur. J. Phys. 26, 985-990 (2005).

[7] Yates, R. C., The Catenary and the Tractrix, Am. Math. Mon. 66, 500-505 (1959).

[8] Cady, W. G., The Circular Tractrix, Am. Math. Mon. 72, 1065-1071 (1965).

[9] Puckette, C. C., The Curve of Pursuit, Math. Gazette 37, 256-260 (1953).

[10] Pijls, W., Some Properties Related to Mercator Projection, Am. Math. Mon. 108, 537-543 (2001).

[11] Bertotti, B., Catenacci, R. and Dappiaggi, C., Pseudospheres in geometry and physics: From Beltrami to de Sitter and beyond, Preprint math/0506395.

[12] Lotka, A. J., Contribution to the Mathematical Theory of Capture. I. Conditions for Capture, Proc. Nat. Acad. Sci. 18, 172-178 (1932).

[13] Lalan, V., Contribution á l"etude de la courbe de poursuite, Comptes Rendus 192, 466-469 (1931).

[14] Lotka, A. J., Families of curves of pursuit, and their isochrones, Am. Math. Mon. 35, 421-424 (1928).

[15] Qing-Xin, Y. and Yin-Xiao, D., Note on the dog-andrabbit chase problem in introductory kinematics, Eur. J. Phys. 29, N43-N45 (2008).

[16] Wunderlich, W., Über die Hundekurven mit konstanten Schielwinkel, Monatshefte für Mathematik 61, 277-331 (1957). 